\title{
Trastuzumab increases the sensitivity of HER2-amplified human gastric cancer cells to oxaliplatin and cisplatin by affecting the expression of telomere-associated proteins
}

\author{
YONGPING LIU ${ }^{1,2^{*}}$, YANG LING ${ }^{2 *}$, QIUFENG QI ${ }^{1}$, MING ZHU $^{1}$, \\ MEIZHEN WAN ${ }^{3}$, YAPING ZHANG ${ }^{1}$ and CHANGSONG ZHANG $^{1}$ \\ ${ }^{1}$ Clinical Oncology Laboratory; Departments of ${ }^{2}$ Oncology Medicine and ${ }^{3}$ Pathology, \\ Changzhou Tumor Hospital Affiliated to Suzhou University, Changzhou, Jiangsu 213002, P.R. China
}

Received August 7, 2014; Accepted November 21, 2014

DOI: $10.3892 / \mathrm{ol} .2014 .2793$

\begin{abstract}
HER2 amplification occurs in $20 \%$ of gastric cancer (GC) cases; however, in gastric and gastroesophageal junction cancer with HER2 gene amplification, trastuzumab in combination with cisplatin (DDP)-based chemotherapy has been reported to improve the oncological outcome. The aim of the present study was to evaluate the combined antitumor efficacy of trastuzumab and various platinum agents in GC cells and to elucidate mechanisms that may be involved in the interaction between trastuzumab and the platinum agents. The in vitro chemosensitivity of the GC cells to platinum agents was evaluated using the CellTiter $96^{\circledR}$ AQueous One Solution Cell Proliferation Assay kit. Treatment with $1.0 \mu \mathrm{g} / \mathrm{ml}$ trastuzumab for $48 \mathrm{~h}$ significantly increased the sensitivity of NCI-N87 cells with HER2 amplification to oxaliplatin (Oxa) and DDP. This chemosensitivity was most prominent in the NCI-N87 cells, in which the half maximal inhibitory concentration of Oxa and DDP was decreased to $\sim 3.29$ and 6.91 times, respectively. The apoptotic effect of the platinum agents was evaluated by double-staining the GC cells with Annexin V-fluorescein isothiocyanate and propodium iodide. Consistent with the chemosensitivity analysis, apoptotic analysis indicated that trastuzumab significantly increased Oxa- and DDP-induced apoptosis in the NCI-N87 cells. Furthermore, the mRNA expression levels of various telomere-associated genes was determined by performing quantitative reverse transcription-polymerase chain reactions in a number of GC cell lines,
\end{abstract}

Correspondence to: Dr Yongping Liu, Clinical Oncology Laboratory, Changzhou Tumor Hospital Affiliated to Suzhou University, 1 North Huaide Road, Changzhou, Jiangsu 213002, P.R. China

E-mail: liuyongping026@126.com

*Contributed equally

Key words: trastuzumab, gastric cancer, sensitivity, platinum, telomere-related protein and revealed that trastuzumab (alone and in combination with DDP) may downregulate the mRNA expression levels of the TPP1, TRF1, TRF2, TRF2IP and POT1 genes. However, western blot analysis demonstrated that trastuzumab (alone and in combination with DDP) may significantly downregulate the protein expression levels of telomeric repeat binding factor 2, protection of telomere 1 and TPP1 (formerly known as TINT1, PTOP and PIP). The results of the present study indicate a potential role of low-dose trastuzumab administration for increasing Oxa and DDP sensitivity in HER2-amplified GC cells, possibly via the downregulation of telomere-associated gene expression.

\section{Introduction}

Globally, gastric cancer (GC) is the predominant cause of gastrointestinal cancer and is the second leading cause of cancer-related mortality $(1,2)$. Despite the use of curative resection for the treatment of resectable GC, recurrence rates remain high following surgery. Furthermore, the majority of patients are diagnosed at an advanced stage of GC, at which surgical resection is no longer feasible; although palliative radiotherapy and chemotherapy provide some benefit, the prognosis of advanced GC remains poor (3-6).

Various attempts have been made to improve the objective response rate to $\mathrm{GC}$ treatment, including the development of biologically targeted agents in combination with traditional chemotherapy regimens; for example, trastuzumab in combination with a cisplatin (DDP)-based chemotherapy regimen. Trastuzumab is a humanized monoclonal anti-human epidermal growth factor receptor 2 (HER2) antibody treatment. The first phase III, prospective, randomized, multicenter trial to evaluate its efficacy and safety for HER2-positive GC treatment was the Trastuzumab for Gastric Cancer (ToGA) study (7-9). Although trastuzumab (Herceptin ${ }^{\mathrm{TM}}$ ) in combination with a DDP-based chemotherapy regimen produced a significant overall survival benefit, the benefit was modest and the mechanism involved was not clearly addressed.

A number of in vitro and in vivo studies have demonstrated that the administration of trastuzumab in combination with chemotherapeutic agents produces an additive effect, a 
synergistic effect or both in breast cancer (10-13). Furthermore, previous in vitro studies demonstrated that trastuzumab in combination with DDP (14) and doxorubicin (15) produces a synergistic effect in human HER2-overexpressing GC cells; however, the mechanisms of this synergistic anticancer activity are yet to be fully explored.

Chromosome ends consist of specialized structures called telomeres that are critical to chromosome integrity. In vertebrates, telomeres consists of thousands of T2AG3 hexamer repeats; the nucleophilic sites of these repeats are able to react with platinum agents to form DNA adducts, principally at adjacent deoxyguanines ( $\mathrm{GpG})$. The DNA adducts formed are 1,2-intrastrand cross-links, which result in the efficient inhibition of DNA replication, RNA transcription, cell cycle arrest or apoptosis. Subject to the presence of two or more tandem guanines, platinum agents exhibit maximal targeting of the DNA; thus, telomeric repeats are a good target for platinum agents (16). Furthermore, a previous in vitro study demonstrated that telomere dysfunction may increase DDP sensitivity in melanoma cells (17) and an increasing number of proteins have been discovered to interact with telomere DNA repeats; for example, telomere protection, function, and length appear to depend on the shelterin protein complex [telomeric repeat binding factor 1 (TRF1), TRF2, TPP1 (formerly known as TINT1, PTOP and PIP), protection of telomere 1 (POT1), TRF1-interacting nuclear factor (TIN2), and TRF2-interacting protein 1 (TRF2IP)] (18). The ToGA study indicated that trastuzumab in combination with a DDP-based chemotherapy regimen resulted in a significant overall survival benefit (9). In the present study, we hypothesize that trastuzumab may additionally affect the expression levels of the abovementioned telomere-associated proteins and, thus, the sensitivity of GC cells to platinum agents.

The present preclinical study was undertaken to investigate the effect of low-dose trastuzumab on the sensitivity of GC cells to platinum agents, and to elucidate the possible mechanisms involved in the interaction between the trastuzumab and platinum agents. In addition, the protein and mRNA expression levels of telomere-associated genes and proteins was investigated in GC cells following treatment with trastuzumab and platinum agents, alone and in combination.

\section{Methods}

Cell lines and cell culture. The effects of trastuzumab, Oxa, DDP, 5-fluorouracil (FU) and taxol administration (alone and in combination) on malignant cell growth were studied in a panel of five human GC cell lines (AGS, NCI-N87, MGC-803, HGC and MKN45) obtained from the Shanghai Institute of Cell Biology (Shanghai, China). Of these, NCI-N87 is an HER2-amplified cell line (19). All of the cell lines were cultured in RPMI-1640 medium (Gibco Life Technologies, Carlsbad, CA, USA) supplemented with $10 \%$ bovine serum (Invitrogen Life Technologies, Carlsbad, CA, USA), $100 \mathrm{mg} / \mathrm{ml}$ streptomycin (Sichuan Pharmaceutical Co., Ltd., Sichuan, China), $100 \mathrm{U} / \mathrm{ml}$ penicillin (Sichuan Pharmaceutical Co., Ltd.), insulin (Tonghua Dongbao Pharmaceutical Co., Ltd., Jilin, China), glutamine and pyruvate (Invitrogen Life Technologies) at $37^{\circ} \mathrm{C}$ in a $5 \% \mathrm{CO}_{2}$ water-saturated atmosphere.
Platinum agents. Prior to each experiment, the dilutions of all of the reagents were freshly prepared. Trastuzumab was obtained from the University of California Pharmaceutical Services (Los Angeles, CA, USA) and was prepared from a stock concentration of $20 \mathrm{mg} / \mathrm{ml}$. Oxa, DDP, 5-FU and Taxol were supplied by the Jiangsu Hengrui Medicine Co., Ltd. (Lianyungang, China). A CellTiter $96^{\circledR}$ AQueous One Solution Cell Proliferation Assay kit was purchased from Promega Corporation (Madison, WI, USA) and the Annexin-V-fluorescein isothiocyanate (FITC) Apoptosis Detection kit was purchased from Invitrogen Life Technologies.

Cell viability assay. The CellTiter 96 kit (Promega Corporation) was used to determine cytotoxicity, according to the manufacturer's instructions. Briefly, the five human GC cell lines were grown to the log phase, trypsinized, seeded into 96-well plates at a density of $2 \times 10^{3}$ cells/well and incubated overnight to allow cell adherence. Subsequently, the medium in each well was replaced with fresh (platinum agent-free) medium or medium containing various concentrations $\left(1 \times 10^{-4}, 1 \times 10^{-3}, 1 \times 10^{-2}\right.$, $1 \times 10^{-1}, 1,10$ and $100 \mu \mathrm{g} / \mathrm{ml}$ ) of platinum agents (Oxa and DDP) and was incubated for an additional $48 \mathrm{~h}$. CellTiter 96 AQueous One solution was added to each well at one fifth of the mixture volume and the plates were incubated for $3 \mathrm{~h}$. Absorbance was determined at an wavelength of $490 \mathrm{~nm}$ using a microplate reader (Bio-Rad Laboratories, Hercules, CA, USA), with blank control wells to zero the absorbance. For each experiment, 10 control wells were allocated for platinum agent-free medium and a minimum of six replicate wells were allocated for each concentration of platinum agent-containing medium. Using the background-corrected absorbance values, the inhibition rate $[\mathrm{I}(\%)]$ was calculated by the following equation: $\mathrm{I}(\%)=100 \mathrm{x}\left(\mathrm{A}_{\text {untreated control well }}-\mathrm{A}_{\text {experimental well }}\right) / \mathrm{A}_{\text {untreated control well }}$. The half maximal inhibitory concentration $\left(\mathrm{IC}_{50}\right)$ was defined as the concentration of platinum agent required for $50 \%$ inhibition of cell growth.

Apoptosis assay. The number of apoptotic cells was quantified using an Annexin V-FITC Apoptosis Detection kit (Invitrogen Life Technologies), according to the manufacturer's instructions. Briefly, the NCI-N87 cells were grown to $75-80 \%$ confluence in 60-mm Petri dishes, exposed to trastuzumab $(1.0 \mu \mathrm{g} / \mathrm{ml})$ and platinum agents (Oxa, $5 \mu \mathrm{g} / \mathrm{ml}$; DDP, $2.5 \mu \mathrm{g} / \mathrm{ml})$ alone or in combination for $48 \mathrm{~h}$, and compared with the untreated control cells. To quantify the apoptosis, the cells were collected, resuspended in $500 \mu \mathrm{l}$ binding buffer, treated with $5 \mu \mathrm{l}$ Annexin V-FITC and $5 \mu \mathrm{l}$ propidium iodide (PI), and analyzed using a FACSCalibur ${ }^{\mathrm{TM}}$ flow cytometer (BD Biosciences, Franklin Lakes, NJ, USA).

Reverse transcription-quantitative polymerase chain reaction $(R T-q P C R)$. Briefly, the three human GC cell lines, NCI-N87, HGC27 and MKN45, were grown to the log phase, trypsinized, washed with phosphate-buffered saline (PBS) and collected by performing centrifugation for $5 \mathrm{~min}$ at 174 x g. Total RNA was extracted from each cell line using the SV Total RNA isolation system (Promega Corporation), according to the manufacturer's instructions. The purity and quality of the extracted mRNA were determined using a Bio-visible spectrophotometer at 260 and $280 \mathrm{~nm}$ 
Table I. Effects of trastuzumab on the $\mathrm{IC}_{50}$ of DDP and Oxa in five gastric cancer cell lines.

Gastric cancer cell line $\left(\mathrm{IC}_{50}\right.$, mean \pm standard deviation $)$

\begin{tabular}{lcccrr}
\cline { 2 - 5 } Agent & NCI-N87 & HGC27 & MGC803 & AGS & MKN45 \\
\hline DDP & $12.86 \pm 1.41$ & $10.23 \pm 1.94$ & $15.26 \pm 1.85$ & $9.39 \pm 1.77$ & $26.44 \pm 2.72$ \\
DDP (trastuzumab) & $1.86 \pm 0.55^{\mathrm{c}}$ & $10.97 \pm 2.36$ & $14.38 \pm 1.63$ & $10.19 \pm 3.02$ & $25.44 \pm 4.70$ \\
Oxa $_{\text {Oxa (trastuzumab) }}{ }^{\mathrm{b}}$ & $21.53 \pm 1.96$ & $15.07 \pm 3.30$ & $27.26 \pm 3.39$ & $19.21 \pm 1.85$ & $35.77 \pm 3.82$ \\
& $6.53 \pm 1.10^{\mathrm{d}}$ & $14.66 \pm 2.66$ & $26.69 \pm 4.47$ & $20.39 \pm 3.70$ & $37.64 \pm 6.66$
\end{tabular}

${ }^{\mathrm{a}}$ Overexpressing HER2/neu gene; ${ }^{\mathrm{b}}$ pretreatment with trastuzumab for $48 \mathrm{~h} ;{ }^{\mathrm{c}} \mathrm{P}=0.002$, vs. DDP group; ${ }^{\mathrm{d}} \mathrm{P}=0.001$, vs. Oxa group. $\mathrm{IC}_{50}$, half maximal inhibitory concentration; DDP, cisplatin; Oxa, oxaliplatin.

(Eppendorf, Hamburg, Germany); and the integrity of the extracted mRNA was determined by performing agarose gel electrophoresis on a $1 \%$ gel . Reverse transcriptase from the reverse transcription system was used according to the manufacturer's instructions (Promega Corporation) to synthesize a total volume of $20 \mu \mathrm{l}$ complementary DNA for each cell line, and the $\mathrm{iCycler} \mathrm{iQ}^{\mathrm{TM}}$ Multi-Color Real Time PCR detection system (Bio-Rad Laboratories, Inc.) was used to perform RT-qPCR of the target genes and the internal control $(\beta$-actin). Applied Biosystems Life Technologies (Foster city, CA, USA) supplied the primers [1X; Assay IDs: Hs00819517_mH (TRF1); Hs01554305_g1 (TIN2); Hs00194619_m1 (TRF2); Hs00368526_g1 (TPP1); Hs00430292_m1 (TRF2IP); Hs00209984_m1 (POT1); and Hs99999903_m1 ( $\beta$-actin)] and probe mixture, and AbGene Ltd. (Surrey, UK) supplied the ABsolute qPCR mix (1X) used to produce the $20-\mu 1 \mathrm{PCR}$ reaction mixture. The PCR conditions were as follows: $50^{\circ} \mathrm{C}$ for $2 \mathrm{~min}, 95^{\circ} \mathrm{C}$ for $15 \mathrm{~min}$, followed by 45 cycles at $95^{\circ} \mathrm{C}$ for $15 \mathrm{sec}$ and $60^{\circ} \mathrm{C}$ for $1 \mathrm{~min}$. Using $\beta$-actin as an endogenous control and commercial human total RNA samples (Clontech Laboratories, Inc., Mountainview, CA, USA) as calibrators, the relative gene expression levels were quantified according to the comparative $\mathrm{Ct}$ method, employed the formula $2^{-\Delta \Delta \mathrm{Ct}}$ to determine the final results (20). Following PCR, the $10-\mu 1$ product was loaded onto a $1.5 \%$ agarose gel and visualized by ethidium bromide staining (Sigma-Aldrich, Munich, Germany).

Western blot analysis. Total cell lysates were prepared in RIPA lysis buffer (Pierce Biotechnology, Inc., Rockford, IL, USA). Following determination of protein concentration using a bicinchoninic acid protein assay kit (Pierce Biotechnology, Inc.), an aliquot of lysate containing $50 \mu \mathrm{g}$ of each protein was subjected to sodium dodecyl sulfate-polyacrylamide gel electrophoresis, transferred to polyvinylidene fluoride membranes, blocked with blocking buffer (PBS Tween-20 containing 5\% non-fat milk) for $2 \mathrm{~h}$ at room temperature and incubated overnight at $4^{\circ} \mathrm{C}$ with the following specific primary antibodies: monoclonal mouse anti-human TPP1 (ACD; cat. no. TA504406), monoclonal rabbit anti-human POT1 (cat. no. TA310771), polyclonal rabbit anti-human TRF1 (cat. no. TA322887), rabbit anti-human monoclonal TRF2 (cat. no. TA307200), rabbit anti-human polyclonal TIN2 (cat. no. TA315321), rabbit anti-human polyclonal TRF2IP (cat. no. TA324532 ) and rabbit anti-human polyclonal GAPDH

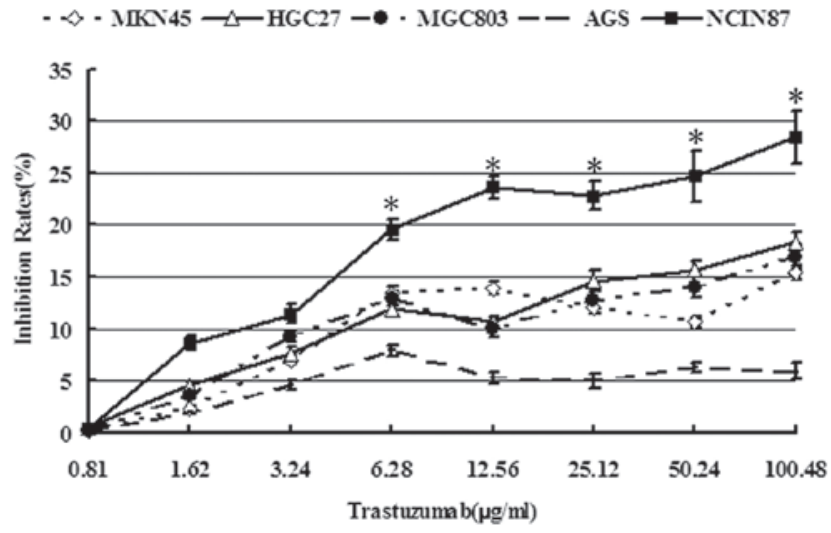

Figure 1. Trastuzumab caused significantly more cytotoxicity to the NCI-N87 cell line with HER2 amplification compared with the other four gastric cancer cell lines investigated. ${ }^{*} \mathrm{P}<0.05$ vs. MKN45, HGC27, MGC-803 and AGS cell lines.

(cat. no. TA308884) (1:10,000, all primary antibodies; OriGene Technologies, Inc., Rockville, MD, USA). Subsequent incubation with the appropriate horseradish peroxidase-conjugated goat anti-rabbit POT1, TRF1, TRF2, TIN2, TRF2IP, GAPDH (cat. no. SP 9001) and goat anti-mouse TPP1 (cat. no. SP9002) secondary antibodies (1:1,000; Santa Cruz Biotechnology, Inc., Santa Cruz, CA, USA) was performed for $2 \mathrm{~h}$ at room temperature. Signals were then detected using enhanced chemiluminescence reagents (Thermo Fisher Scientific, Waltham, MA, USA), and a FluorChem SP imaging system (Alpha Innotech, San Leandro, CA, USA) was used for image capture.

Statistical analysis. Values are expressed as the mean \pm standard deviation. All statistical analyses were performed using SPSS version 13.0 software (SPSS, Inc., Chicago, IL, USA). Statistical comparison was performed using Student's t-test and $\mathrm{P}<0.05$ was considered to indicate a statistically significant difference.

\section{Results}

Trastuzumab renders HER2-amplified cancer cells sensitive to platinum agents. The cytotoxicity of trastuzumab to five GC cell lines was initially analyzed using a CellTiter 96 Aqueous One Solution Cell Proliferation Assay kit. It was identified 

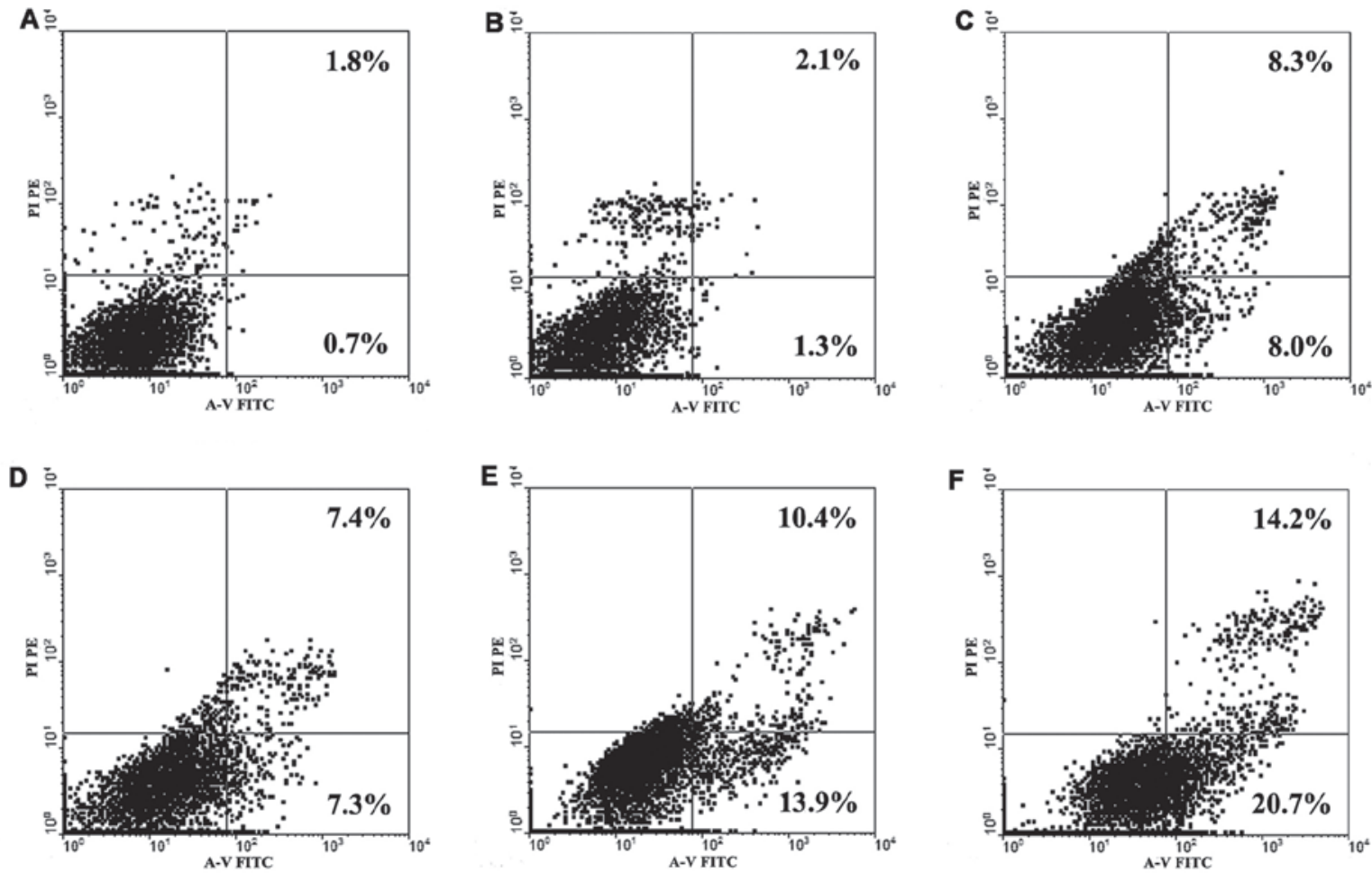

Figure 2. Trastuzumab treatment increases oxaliplatin- and cisplatin-induced apoptosis in NCI-N87 cells. (A) Untreated cells, and cells treated with (B) trastuzumab alone, (C) oxaliplatin alone, (D) cisplatin alone, (E) trastuzumab in combination with oxaliplatin and (F) trastuzumab in combination with cisplatin. A-V FITC, Annexin-V fluorescein isothiocyanate; PI PE, propidium iodide phycoerythrin.

that trastuzumab $(6.28,12.56,25.12,50.24$ and $100.48 \mu \mathrm{g} / \mathrm{ml})$ rendered more significant cytotoxicity to the NCI-N87 cell line with HER2 amplification compared with the four other cell lines $(\mathrm{P}<0.05)$. At $0.81-100.48 \mu \mathrm{g} / \mathrm{ml}$ trastuzumab, the inhibition rates to NCI-N87 cells were not $>30 \%$ and $0.81-1.62 \mu \mathrm{g} / \mathrm{ml}$ trastuzumab was not obviously cytotoxic to cancer cells (survival rate, $>90 \%$; Fig. 1). Thus, as treatment of the cells with $1.0 \mu \mathrm{g} / \mathrm{ml}$ trastuzumab exhibited no significant effect on cell viability, this concentration was used for subsequent analyses.

Additionally, $1.0 \mu \mathrm{g} / \mathrm{ml}$ trastuzumab was administered to five GC cell lines and the effect on the sensitivity of the cell lines to various platinum agents was investigated. It was identified that pretreatment with trastuzumab significantly increased the sensitivity of only the NCI-N87 cell line to platinum agents. As indicated in Table $\mathrm{I}$, the $\mathrm{IC}_{50}$ of Oxa and DDP was decreased to $2.29(\mathrm{P}=0.001)$ and 6.91 times $(\mathrm{P}=0.002)$ in NCI-N87 cells, respectively; however, trastuzumab did not alter the $\mathrm{IC}_{50}$ of Oxa or DDP in the other four cell lines. Subsequently, it was identified that simultaneous treatment with low-dose trastuzumab and platinum agents may significantly increase the sensitivity of NCI-N87 cells to platinum agents; for example, the $\mathrm{IC}_{50}$ of Oxa and DDP were reduced by $\sim 2.67(\mathrm{P}=0.001)$ and 4.56 times $(\mathrm{P}=0.003)$, respectively. Thus, these data indicate that low-dose trastuzumab may increase platinum sensitivity in NCI-N87 cells.

Trastuzumab treatment increases platinum agent-induced apoptosis in NCI-N87 cells. To further investigate whether low-dose trastuzumab increases the sensitivity of NCI-N87 cells to platinum agents, GC cells were double-stained with Annexin V-FITC and PI to detect early apoptosis induced by treatment with platinum agents and trastuzumab, alone or in combination. The doses of Oxa and DDP selected were $5 \mu \mathrm{g} / \mathrm{ml}$ and $2.5 \mu \mathrm{g} / \mathrm{ml}$, respectively, as they were close to the $20 \%$ inhibitory concentrations $\left(\mathrm{IC}_{20}\right)$ of NCI-N87 cells. The dose of trastuzumab used was $1.0 \mu \mathrm{g} / \mathrm{ml}$, as previously determined. For the groups treated with two agents in combination, the cells were pretreated with trastuzumab for $48 \mathrm{~h}$, followed by the addition of platinum agents for $48 \mathrm{~h}$. As indicated in Fig. 2, the percentage of early apoptosis induced by independent Oxa and DDP administration in NCI-N87 cells was 8.0 and $7.3 \%$, respectively; however, this increased to 13.9 and $20.7 \%$ upon treatment with trastuzumab in combination with Oxa and DDP, respectively. Furthermore, treatment with trastuzumab alone $(1.0 \mu \mathrm{g} / \mathrm{ml})$ did not induce significant apoptosis in the cancer cells. Thus, it appears that low-dose trastuzumab administration may increase platinum agent-induced apoptosis in NCI-N87 cells and subsequently result in the increased cytotoxicity of platinum agents (Fig. 1).

Trastuzumab downregulates $m R N A$ and protein expression levels of telomere-associated genes in NCI-N87 cells. To understand the mechanisms by which trastuzumab may increase the sensitivity of NCI-N87 cells to platinum agents, we hypothesized that trastuzumab may affect the expression levels of telomere-associated genes or proteins in NCI-N87 cells, thus, influencing their sensitivity to platinum agents. Following incubation with $1.0 \mu \mathrm{g} / \mathrm{ml}$ trastuzumab alone, $2.5 \mu \mathrm{g} / \mathrm{ml}$ DDP alone or the two agents in combination, the mRNA expression of various telomere-associated genes and their proteins were assessed in NCI-N87 cells by performing RT-qPCR and western blot analysis. As demonstrated in Fig. 3A, the mRNA 


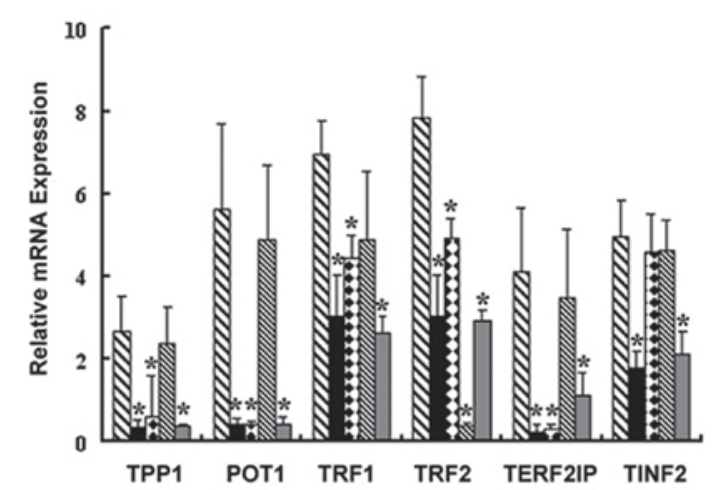

B

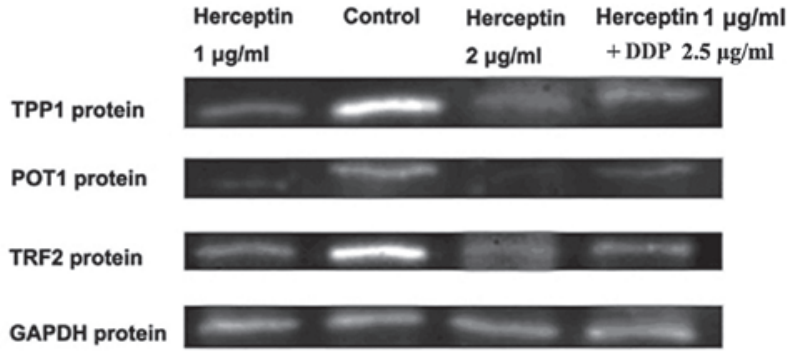

Figure 3. Effect of trastuzumab administration on the expression of telomere-associated genes and proteins in NCI-N87 cells. (A) Reverse transcription-quantitative polymerase chain reaction was used to determine the levels of TPP1, POT1, TRF1,TRF2, TERF2IP and TINF2 mRNA expression in NCI-N87 cells treated with trastuzumab and cisplatin (alone or in combination). Error bars indicate the standard deviation from the mean. ${ }^{*} \mathrm{P}<0.05$, vs. control cells. (B) Western blot analysis determined that the protein expression levels of TPP1, POT1 and TRF2 in NCI-N87 cells were significantly inhibited by various concentrations of trastuzumab alone and low-dose trastuzumab in combination with cisplatin. TPP1 (formerly known as TINT1, PTOP and PIP); POT1, protection of telomere 1; TRF2, telomeric repeat binding factor 2; Control, untreated NCI-N87 cells.

expression levels of TRF1, TRF2, POT1, TPP1 and TRF2IP were significantly downregulated following treatment with trastuzumab alone or DDP in combination with trastuzumab in NCI-N87 cells $(\mathrm{P}<0.05)$, and DDP alone only downregulated the expression level of TRF2 $(\mathrm{P}<0.05)$. However, in the HGC27 and MKN45 cell lines, treatment wth low dose trastuzumab alone or in combination with DDP did not significantly downregulate the mRNA expression levels of any of the telomere-associated genes $(\mathrm{P}>0.05)$ (data not shown). Western blot analysis indicated that treatment with trastuzumab alone and in combination with DDP significantly downregulated the protein expression levels of TRF2, POT1 and TPP1 in NCI-N87 cells, and DDP alone did not downregulate the protein expression level of any of the telomere-associated genes investigated (data not shown). The results indicated that low-dose trastuzumab administration may downregulate the mRNA and protein expression levels in an area of the telomere-associated genes that may be involved in chemosensitivity NCI-N87 cells to platinum agents.

\section{Discussion}

Overexpression and activation of $H E R 2$ has previously been associated with chemotherapy resistance in HER2 gene-amplified cancer cells (21-23), and telomere dysfunction has been associated with platinum sensitivity in cancer cells (17). The aim of the present study was to investigate whether the antitumor activity of platinum agents commonly used in the treatment GC could be enhanced by the addition of low-dose trastuzumab and to discuss the possible mechanisms of platinum agent-resistance in GC cells, which may involve telomere-associated proteins. First, it was investigated whether low-dose trastuzumab could increase the sensitivity of five GC cell lines to platinum agents by performing cell proliferation and early apoptosis analysis. The findings of the present study indicate that treatment with low-dose trastuzumab may reduce the $\mathrm{IC}_{50}$ of platinum agents and increase the early apoptosis rates induced by platinum agents in NCI-N87 cells, which overexpress HER2 gene; however, this increase in sensitivity was not observed in the other four cell lines. Additionally, the expression levels of telomere-associated genes appeared to be downregulated in NCI-N87 cells following treatment with low-dose trastuzumab alone or trastuzumab in combination with DDP. These results indicate that pretreatment with low-dose trastuzumab followed by platinum agent administration may be a promising method the treatment of HER2-positive advanced GC. Thus, a possible, partial mechanism for the increase in platinum agent sensitivity may be the downregulation of the expression of telomere-related genes.

HER-2/neu or c-erbB-2 is a member of the HER family of growth factors (endothelial growth factor receptor, erbB-2, erbB-3 and erbB-4), which exhibit intrinsic protein tyrosine kinase activity. Increased HER-2 activity is the assumed mechanism underlying cell cycle control, proliferation, differentiation, motility, apoptosis, metastasis and transformation (14,24-26) and HER-2 overexpression is observed in numerous human carcinomas, including breast, ovarian, gastric, colon and non-small cell lung cancer. Trastuzumab, a humanized monoclonal antibody (mAb) against the extracellular domain of HER2, has been approved by the Food and Drug Administration for the treatment of patients with invasive HER2-overexpressing breast cancer (27). Additionally, HER2-amplified GC is the most probable candidate for responding to trastuzumab treatment, as various studies have demonstrated that an antitumor effect occurs when trastuzumab is added to HER2-amplified GC cells or the corresponding xenograft models $(19,28,29)$. Additionally, the results of the present study demonstrated significant cytotoxicity of trastuzumab on the HER2-amplified NCI-N87 cell line; however, the inhibition rates were not $>30 \%$, even at the highest concentration. These results are consistent with those of a previous in vitro study (30), which indicated that the administration of trastuzumab alone may not exhibit a significant antitumor effect.

Numerous in vitro and in vivo studies have demonstrated that additive and synergistic effects occur when trastuzumab is combined with other chemotherapeutic agents to treat breast cancer and GC (10-14). The mechanisms by which this occurs include a reduction in DNA repair activity following chemotherapeutic agent-induced DNA damage $(31,32)$, inhibition of the unscheduled DNA synthesis (33), and increased apoptosis via the activation of antibody responses and reduced expression of anti-apoptotic genes $(34,35)$. 
In the present study, it was identified that low-dose trastuzumab administration may significantly decrease the $\mathrm{IC}_{50}$ of platinum agents and increase the early apoptosis rates induced by them in NCI-N87 cells, which overexpress the HER2 gene, but not in other four cell lines. These results indicated that the HER2/neu signaling pathway may participate in the mechanisms of platinum agent chemosensitivity in HER2-amplified GC. The results obtained in the present study are partially consistent with those reported by Yu and Hung (36), Funato et al (37), and Järvinen and Liu (38). For example, Yu and Hung (36) reported that the trastuzumab-induced increase in paclitaxel sensitivity in HER2-overexpressing breast cancer cells may occur by reversing the anti-apoptotic function of HER2. In the present study, the administration of trastuzumab alone at $1.0 \mu \mathrm{g} / \mathrm{ml}$ did not induce significant apoptosis in the NCI-N87 cells; however, when the NCI-N87 cells were treated with trastuzumab in combination with DDP or Oxa for $48 \mathrm{~h}$, the early apoptotic rates were markedly increased to 20.7 and $13.9 \%$, respectively. Thus, it appears that trastuzumab may increase platinum agent-induced apoptosis in NCI-N87 cells, resulting in the increased cytotoxicity of platinum agents.

Telomeres are nucleoprotein complexes located at the ends of chromosomes. In vertebrates, telomeres consists of tandem repeats of the T2AG3 hexamer, a G-rich motif and associated proteins (39). Due to the presence of guanine triplets, telomeric DNA is considered to be a preferential target for DDP (16). Furthermore, telomeres are critical for genomic stability as they provide a mechanism for the maintenance and protection of chromosomal ends by folding into a structure termed the T-loop. The T-loop is characterized by a single-stranded overhang of 30 nucleotides, which is sequestered by invasion of a duplex region of the telomere (39). TRF1, TRF2, POT1, TRF2IP, TPP1 and TIN2 form a six-protein complex termed shelterin (18), which contributes to the formation of the T-loop as well as the telomere protection and length regulation. Within shelterin, TRF1, TRF2 and POT1 bind directly to the telomeric DNA; TRF1 forms a complex with a number of proteins, including tankyrase, TIN2, TPP1 and POT1 (40-43); and TRF2 interacts with various proteins, including TRF2IP, TIN2 and POT1 (41,42,44-46). The uncapping of telomeres due to dysfunctional telomeric proteins or telomere shortening can disrupt their protective function and activate a DNA damage response $(47,48)$. The loss of telomere protection, whether induced by telomere shortening or via the disruption of telomere structure, is commonly referred to as telomere dysfunction, and has been reported as the principal determinant governing chemosensitivity against agents that induce double-strand DNA breaks (49,50).

In conclusion, the present study identified that the mRNA and protein expression levels of TRF2, POT1 and TPP1 were significantly downregulated following treatment with trastuzumab alone or trastuzumab in combination with DDP. Thus, it is proposed that the HER2/neu signaling pathway modulates the expression of a number of telomere-associated proteins, resulting in telomere dysfunction. Telomere dysfunction may represent a physiological trigger of the DNA damage or apoptotic response, analogously to other genotoxic insults that introduce chromosome breaks. Concurrently, telomere dysfunction may additionally contribute to sensitizing the platinum agents in HER2-amplified GC cells. The mechanisms by which trastuzumab influences the expression of these telomere-associated proteins remain unclear; however, a possible mechanism may involve changes in numerous signal transduction pathways associated with drug-gene or drug-protein interactions. Thus, additional studies are required to analyze the effects of platinum agents on the gene and protein expression profiles of various signaling pathways.

\section{Acknowledgements}

The abstract was presented at the 2013 Gastrointestinal Cancers Symposium and published as abstract no. 53 in J Clin Oncol 31 (suppl 4; abstr 53), 2013. The present authors thank the personnel at the Changzhou Tumor Hospital (Changzhou, China) who were involved in the present study. The current study was supported by the Science and Technology Planning Project of Changzhou Health Bureau (Jiangsu, China; grant nos. QN201106 and ZD201203), the Natural Science Foundation of China (grant nos. 81071799, 81372212, BK2011251 and BL2013012), the Research Project of the Health Department of Jiangsu Province (grant no. Z201221), the Science and Technology Planning Project of Jiangsu (grant no. CE20135051), the Science and Technology Planning Project of Traditional Chinese Medicine, Jiangsu (grant no. LZ13143), the 333 Talents Training Project of Jiangsu Province (grant no. 2011-0635), the Key Medical Innovation Talents Training Project of Changzhou (grant no. 2010368), and the Project of Jiangsu Province Sanitation Innovation Team (grant no. LJ201157).

\section{Reference}

1. Leung WK, Wu MS, Kakugawa Y, et al; Asia Pacific Working Group on Gastric Cancer: Screening for gastric cancer in Asia: current evidence and practice. Lancet Oncol 9: 279-287, 2008.

2. Kamangar F, Dores GM and Anderson WF: Patterns of cancer incidence, mortality, and prevalence across five continents: defining priorities to reduce cancer disparities in different geographic regions of the world. J Clin Oncol 24: 2137-2150, 2006.

3. Hundahl SA, Phillips JL and Menck HR: The National Cancer Data Base Report on poor survival of U.S. gastric carcinoma patients treated with gastrectomy: Fifth Edition American Joint Committee on Cancer staging, proximal disease, and the "different diseas"' hypothesis. Cancer 88: 921-932, 2000.

4. Cunningham D, Allum WH, Stenning SP, Thompson JN, Van de Velde CJ, Nicolson M, Scarffe JH, Lofts FJ, Falk SJ, Iveson TJ, et al; MAGIC Trial Participants: Perioperative chemotherapy versus surgery alone for resectable gastroesophageal cancer. New Engl J Med 355: 11-20, 2006.

5. Macdonald JS, Smalley SR, Benedetti J, Hundahl SA, Estes NC, Stemmermann GN, Haller DG, Ajani JA, Gunderson LL, Jessup JM and Martenson JA: Chemoradiotherapy after surgery compared with surgery alone for adenocarcinoma of the stomach or gastroesophageal junction. New Engl J Med 345: 725-730, 2001.

6. Smalley SR, Benedetti JK, Haller DG, Hundahl SA, Estes NC, Ajani JA, Gunderson LL, Goldman B, Martenson JA, Jessup JM, et al: Updated analysis of SWOG-directed intergroup study 0116: a phase III trial of adjuvant radiochemotherapy versus observation after curative gastric cancer resection. J Clin Oncol 30: 2327-2333, 2012.

7. Hede K: Gastric cancer: trastuzumab trial results spur search for other targets. J Natl Cancer Inst 101: 1306-1307, 2009.

8. Jørgensen JT: Targeted HER2 treatment in advanced gastric cancer. Oncology 78: 26-33, 2010.

9. Bang YJ, Van Cutsem E, Feyereislova A, et al; ToGA Trial Investigators: Trastuzumab in combination with chemotherapy versus chemotherapy alone for treatment of HER2-positive advanced gastric or gastro-oesophageal junction cancer (ToGA): a phase 3, open-label, randomised controlled trial. Lancet 376: 687-697, 2010. 
10. Pegram MD, Konecny GE, O'Callaghan C, Beryt M, Pietras R and Slamon DJ: Rational combinations of trastuzumab with chemotherapeutic drugs used in the treatment of breast cancer. J Natl Cancer Inst 96: 739-749, 2004.

11. Pegram M, Hsu S, Lewis G, et al: Inhibitory effects of combinations of HER-2/neu antibody and chemotherapeutic agents used for treatment of human breast cancers. Oncogene 18: 2241-2251, 1999

12. Pegram MD, Pienkowski T, Northfelt DW, et al: Results of two open-label, multicenter phase II studies of docetaxel, platinum salts, and trastuzumab in HER2-positive advanced breast cancer. J Natl Cancer Inst 96: 759-769, 2004.

13. Robert N, Leyland-Jones B, Asmar L, et al: Randomized phase III study of trastuzumab, paclitaxel, and carboplatin compared with trastuzumab and paclitaxel in women with HER-2-overexpressing metastatic breast cancer. J Clin Oncol 24 2786-2792, 2006.

14. Kim SY, Kim HP, Kim YJ, et al: Trastuzumab inhibits the growth of human gastric cancer cell lines with HER2 amplification synergistically with cisplatin. Int J Oncol 32: 89-95, 2008.

15. Gong SJ, Jin CJ, Rha SY and Chung HC: Growth inhibitory effects of trastuzumab and chemotherapeutic drugs in gastric cancer cel lines. Cancer Lett 214: 215-224, 2004.

16. Burstyn JN, Heiger-Bernays WJ, Cohen SM and Lippard SJ Formation of cis-diamminedichloroplatinum(II) 1,2-intrastrand cross-links on DNA is flanking-sequence independent. Nucleic Acids Res 28: 4237-4243, 2000.

17. Biroccio A, Gabellini C, Amodei S, et al: Telomere dysfunction increases cisplatin and ecteinascidin-743 sensitivity of melanoma cells. Mol Pharmacol 63: 632-638, 2003.

18. de Lange T: Shelterin: the protein complex that shapes and safeguards human telomeres. Genes Dev 19: 2100-2110, 2005

19. Tanner M, Hollmén M, Junttila TT, et al: Amplification of HER-2 in gastric carcinoma: association with Topoisomerase II $\alpha$ gene amplification, intestinal type, poor prognosis and sensitivity to trastuzumab. Ann Oncol 16: 273-278, 2005.

20. Livak KJ and Schmittgen TD: Analysis of relative gene expression data using real-time quantitative PCR and the $2^{-\Delta \Delta C T}$ method. Methods 25: 402-408, 2001

21. Modi S, DiGiovanna MP, Lu Z, et al: Phosphorylated/activated HER2 as a marker of clinical resistance to single agent taxane chemotherapy for metastatic breast cancer. Cancer Invest 23 483-487, 2005

22. Tsai CM, Chang KT, Perng RP, et al: Correlation of intrinsic chemoresistance of non-small-cell lung cancer cell lines with HER-2/neu gene expression but not with ras gene mutations. J Natl Cancer Inst 85: 897-901, 1993.

23. Calikusu Z, Yildirim Y, Akcali Z, et al: The effect of HER2 expression on cisplatin-based chemotherapy in advanced non-smal cell lung cancer patients. J Exp Clin Cancer Res 28: 97, 2009

24. Klapper LN, Glathe S, Vaisman N, et al: The ErbB-2/HER2 oncoprotein of human carcinomas may function solely as a shared coreceptor for multiple stroma-derived growth factors. Proc Nat Acad Sci USA 96: 4995-5000, 1999.

25. Moasser MM: Targeting the function of the HER2 oncogene in human cancer therapeutics. Oncogene 26: 6577-6592, 2007.

26. Hung MC and Lau YK: Basic science of HER-2/neu: a review. Semin Oncol 26 (Suppl 12): 51-59, 1999.

27. Hudis CA: Trastuzumab - mechanism of action and use in clinical practice. N Engl J Med 357: 39-51, 2007.

28. Matsui Y, Inomata M, Tojigamori M, Sonoda K, Shiraishi N and Kitano S: Suppression of tumor growth in human gastric cancer with HER 2 overexpression by an anti-HER 2 antibody in a murine model. Int J Oncol 27: 681-685, 2005.

29. Fujimoto-Ouchi K, Sekiguchi F, Yasuno H, Moriya Y, Mori K and Tanaka Y: Antitumor activity of trastuzumab in combination with chemotherapy in human gastric cancer xenograft models. Cancer Chemother Pharmacol 59: 795-805, 2007.
30. Wainberg ZA, Anghel A, Desai AJ, et al: Lapatinib, a dual EGFR and HER2 kinase inhibitor, selectively inhibits HER2-amplified human gastric cancer cells and is synergistic with trastuzumab in vitro and in vivo. Clin Cancer Res 16: $1509-1519,2010$

31. Pietras RJ, Fendly BM, Chazin VR, Pegram MD, Howell SB and Slamon DJ: Antibody to HER-2/neu receptor blocks DNA repair after cisplatin in human breast and ovarian cancer cells. Oncogene 9: 1829-1838, 1994.

32. Boone JJ, Bhosle J, Tilby MJ, Hartley JA and Hochhauser D: Involvement of the HER2 pathway in repair of DNA damage produced by chemotherapeutic agents. Mol Cancer Ther 8: 3015-3023, 2009.

33. Pietras RJ, Pegram MD, Finn RS, Maneval DA and Slamon DJ: Remission of human breast cancer xenografts on therapy with humanized monoclonal antibody to HER-2 receptor and DNA-reactive drugs. Oncogene 17: 2235-2249, 1998.

34. Clynes RA, Towers TL, Presta LG and Ravetch JV: Inhibitory Fc receptors modulate in vivo cytotoxicity against tumor targets. Nat Med 6: 443-446, 2000.

35. Grazette LP, Boecker W, Matsui T, et al: Inhibition of ErbB2 causes mitochondrial dysfunction in cardiomyocytes: implications for herceptin-induced cardiomyopathy. J Am Coll Cardiol 44: 2231-2238, 2004.

36. Yu D and Hung MC: Role of erbB2 in breast cancer chemosensitivity. Bioessays 22: 673-680, 2000.

37. Funato T, Kozawa K, Fujimaki S, Miura T and Kaku M: Increased sensitivity to cisplatin in gastric cancer by antisense inhibition of the her-2/neu (c-erbB-2) gene. Chemotherapy 47 : 297-303, 2001

38. Järvinen TA and Liu ET: Effects of HER-2/neu on chemosensitivity of tumor cells. Drug Resist Updat 3: 319-324, 2000.

39. Griffith JD, Comeau L, Rosenfield S, et al: Mammalian telomeres end in a large duplex loop. Cell 97: 503-514, 1999.

40. Loayza D and De Lange T: POT1 as a terminal transducer of TRF1 telomere length control. Nature 423: 1013-1018, 2003.

41. Houghtaling BR, Cuttonaro L, Chang W and Smith S: A dynamic molecular link between the telomere length regulator TRF1 and the chromosome end protector TRF2. Curr Biol 14: $1621-1631,2004$.

42. Liu D, O'Connor MS, Qin J and Songyang Z: Telosome, a mammalian telomere-associated complex formed by multiple telomeric proteins. J Biol Chem 279: 51338-51342, 2004.

43. Ye JZ, Donigian JR, van Overbeek M, et al: TIN2 binds TRF1 and TRF2 simultaneously and stabilizes the TRF2 complex on telomeres. J Biol Chem 279: 47264-47271, 2004.

44. Li B, Oestreich S and de Lange T: Identification of human Rap1: implications for telomere evolution. Cell 101: 471-483, 2000.

45. Kim SH, Beausejour C, Davalos AR, Kaminker P, Heo SJ and Campisi J: TIN2 mediates functions of TRF2 at human telomeres. J Biol Chem 279: 43799-43804, 2004.

46. Yang Q, Zheng YL and Harris CC: POT1 and TRF2 cooperate to maintain telomeric integrity. Mol Cell Biol 25: 1070-1080, 2005.

47. van Steensel B, Smogorzewska A and de Lange T: TRF2 protects human telomeres from end-to-end fusions. Cell 92: 401-413, 1998.

48. Karlseder J, Smogorzewska A and de Lange T: Senescence induced by altered telomere state, not telomere loss. Science 295: 2446-2449, 2002.

49. Wong KK, Chang S, Weiler SR, Ganesan S, Chaudhuri J, Zhu C, Artandi SE, Rudolph KL, Gottlieb GJ, Chin L,et al: Telomere dysfunction impairs DNA repair and enhances sensitivity to ionizing radiation. Nat Genet 26: 85-88, 2000.

50. Lee KH, Rudolph KL, Ju YJ, Greenberg RA, Cannizzaro L, Chin L,Weiler SR and DePinho RA: Telomere dysfunction alters the chemotherapeutic profile of transformed cells. Proc Natl Acad Sci USA 98: 3381-3386, 2001. 